\title{
Hydrogen Desorption Behavior of Pure Iron and Inconel 625 during Elastic and Plastic Deformation
}

\author{
Hiroki SHODA, ${ }^{1)}$ Hiroshi SUZUKI, ${ }^{21,3)}$ Kenichi TAKAI ${ }^{2,3)}$ and Yukito HAGIHARA ${ }^{21}$ \\ 1) Formerly Graduate Student, Sophia University. Now at NSK Ltd., 7-1 Kioi-cho, Chiyoda-ku, Tokyo 102-8554 Japan. \\ 2) Department of Engineering and Applied Science, Faculty of Science and Technology, Sophia University, 7-1 Kioi-cho, \\ Chiyoda-ku, Tokyo 102-8554 Japan. 3) "Fundamental Studies on Technologies for Steel Materials with Enhanced \\ Strength and Functions", Consortium of JRCM (The Japan Research and Development Center for Metals).
}

(Received on May 13, 2009; accepted on September 18, 2009; originally published in Tetsu-to-Hagané, Vol. 95, 2009, No. 7, pp. 573-581)

\begin{abstract}
The hydrogen desorption behavior of pure iron with a body-centered-cubic (BCC) lattice and Inconel 625 with a face-centered-cubic (FCC) lattice was examined during tensile deformation using a quadrupole mass spectrometer in a vacuum chamber integrated with a tensile testing machine. Hydrogen and water desorption was continuously detected simultaneously under the application of a tensile load and strain to the specimens. Hydrogen desorption promoted by tensile deformation can be found by deducting both fragment hydrogen dissociated from $\mathrm{H}_{2} \mathrm{O}$ and $\mathrm{H}_{2}$ desorbed under unloading from the total amount of hydrogen desorbed from hydrogen-charged specimens during tensile deformation. Hydrogen desorption from hydrogencharged specimens was detected under various strain rates of $4.2 \times 10^{-5} / \mathrm{s}, 4.2 \times 10^{-4} / \mathrm{s}$ and $4.2 \times 10^{-3} / \mathrm{s}$. Hydrogen desorption rarely increased under elastic deformation. In contrast, it increased rapidly at the proof stress when plastic deformation began, reached its maximum, and then decreased gradually with increasing applied strain for both pure iron and Inconel 625. This desorption behavior is closely related to hydrogen dragging by moving dislocations. The amount of desorbed hydrogen promoted by tensile deformation was measured by thermal desorption analysis (TDA). The TDA results showed that the amount of desorbed hydrogen differed at each strain rate. The largest amount of desorbed hydrogen promoted by tensile deformation was $16 \%$ of the initial hydrogen content in pure iron with a high hydrogen diffusion rate when the specimen was deformed at a strain rate of $4.2 \times 10^{-4} / \mathrm{s}$. In contrast, that of Inconel 625 with a low hydrogen diffusion rate was $9 \%$ of the initial hydrogen content when the alloy was deformed at a strain rate of $4.2 \times 10^{-6} / \mathrm{s}$. This difference in the amount of desorbed hydrogen transported by dislocations depends on the balance between the hydrogen diffusion rate and mobile dislocation velocity.
\end{abstract}

KEY WORDS: hydrogen; lattice defect; dislocation; pure iron; Inconel 625; elastic deformation; plastic deformation; hydrogen desorption; hydrogen embrittlement.

\section{Introduction}

One of the authors has reported that there is a close relationship between the trapping states of hydrogen in metals and hydrogen embrittlement. ${ }^{1)}$ For example, it has been demonstrated with cold-drawn pearlite steels that a weakly trapped state of hydrogen diffusive at room temperature causes a considerable reduction of fracture strain in spite of a low hydrogen content. In contrast, a strongly trapped state of hydrogen non-diffusive at room temperature is innocuous to hydrogen embrittlement in spite of a high hydrogen content. The trapping sites of this weakly trapped state are dislocations, vacancies, grain boundaries, cementite interfaces, and solid solution hydrogen in the lattice. ${ }^{1-4)}$ However, there is little agreement about the effects of trapping sites and how they cause hydrogen embrittlement. ${ }^{5-8)}$

The role of weakly trapped hydrogen or solid solution hydrogen diffusive at room temperature has been examined for Inconel 625 and pure iron. $\left.{ }^{9}\right)$ The results revealed that plastic straining markedly increased lattice defects in the presence of hydrogen in hydrogen-charged specimens. Since such lattice defects were subsequently removed by annealing at $200^{\circ} \mathrm{C}$, probably most of the lattice defects introduced during straining in the presence of hydrogen were not dislocations but vacancies and their clusters. ${ }^{9,10)}$ In addition, ductility loss did not recover when an enhanced density of lattice defects induced by both straining and weakly trapped diffusive hydrogen still remained in the specimen even though hydrogen was not present in the final stage of fracture. It is to be noted that hydrogen was not necessarily requisite for embrittlement in the final fracture stage. ${ }^{9)}$ These findings demonstrated that an increased density of lattice defects such as vacancies and their clusters introduced by both straining and weakly trapped diffusive hydrogen was a direct factor causing hydrogen embrittlement.

The creation of vacancies during plastic deformation without the presence of hydrogen is one outcome of the dislocation dynamics such as the intersection and cutting of 
screw dislocations or the combination of edge dislocations with opposite characteristics located on slip planes an atomic plane distance apart. ${ }^{11)}$ Since hydrogen stabilizes vacancies, ${ }^{12,13)}$ the formation of vacancies can be promoted during plastic deformation in the presence of hydrogen. Therefore, the interactions between hydrogen and dislocations are important to hydrogen-enhanced strain-induced vacancies. ${ }^{14,15)}$

Interactions between hydrogen and dislocations occur in various ways. Since the effective radius of a hydrogen atmosphere formed around a dislocation is about $0.5 \mathrm{~nm}$, hydrogen is trapped from the core to the elastic field of the dislocation located at some distance from the core. ${ }^{16)}$ Tritium desorption has been reported from various metals during tensile deformation, suggesting that it was transported by moving dislocations. ${ }^{17-21)}$ In addition, the use of a silver decoration technique revealed the deposition of $\operatorname{Ag}(\mathrm{CN})_{2}$ along the slip lines on a pure aluminum surface, implying the rapid transport of hydrogen together with such dislocations. ${ }^{22)}$ Similar results were obtained using a hydrogen microprint technique: silver particles observed along the slip lines on an $\mathrm{Al}-5 \% \mathrm{Mg}$ alloy surface presumably resulted from the transport of hydrogen to the surface by moving dislocations. ${ }^{23)}$ Hydrogen desorbed from various metals during tensile deformation was detected and a hydrogen desorption peak was observed at the time of fracture. ${ }^{24)} \mathrm{Al}-$ though there are a lot of reports concerning hydrogen desorption promoted by deformation, there are few detailed in situ studies on hydrogen desorption from metals during elastic/plastic deformation.

In the present study, hydrogen desorption behavior attributable not to thermal energy but to mechanical energy, i.e., tensile stress, was analyzed at room temperature at which hydrogen embrittlement occurs. The desorption behavior of weakly trapped diffusive hydrogen was analyzed in situ and the effect of elastic/plastic deformation on hydrogen desorption behavior was investigated for pure iron and Inconel 625 , in which a reduction of fracture strain caused by hydrogen was confirmed.

\section{Experimental}

A pure iron with a bcc lattice and a Ni-based alloy, Inconel 625, with an fcc lattice were used. The chemical compositions are shown in Table 1. Flat specimens of $1 \mathrm{~mm}$ in thickness, $20 \mathrm{~mm}$ in gage length and $2.5 \mathrm{~mm}$ in width were used in order to apply strain during tensile testing. Pure iron was annealed at $900^{\circ} \mathrm{C}$ for $0.5 \mathrm{~h}$ in Ar followed by furnace cooling and Inconel 625 was solution-treated at $1150^{\circ} \mathrm{C}$ for $1.5 \mathrm{~h}$ in $\mathrm{Ar}$ followed by water quenching. The surfaces of all the heat-treated specimens were polished with emery papers to remove any residual oxide film.

Hydrogen charging was carried out by cathodic electrolysis using a galvanostat at a current density of $50 \mathrm{~A} / \mathrm{m}^{2}$ for a sufficient period of time for hydrogen to saturate the specimen. Excluding the gage portion, specimens were covered with resin insulation. The specimen was used as a working electrode and platinum was used as a counter electrode. An aqueous solution of $\mathrm{H}_{2} \mathrm{SO}_{4}$ with a $\mathrm{pH}$ of 2.5 was used to which 0.09 mass $\% \mathrm{NH}_{4} \mathrm{SCN}$ was added. The temperature of the electrolyte was $30^{\circ} \mathrm{C}$ for pure iron and $90^{\circ} \mathrm{C}$ for Inconel
Table 1. Chemical compositions of materials used (mass $\%$ ).

\begin{tabular}{|c|c|c|c|c|c|c|}
\hline \multirow{2}{*}{ Pure iron } & $\mathrm{Fe}$ & $\mathrm{C}$ & $\mathrm{Si}$ & $\mathrm{Mn}$ & $\mathrm{O}$ & $\mathrm{N}$ \\
\cline { 2 - 7 } & 99.97 & 0.001 & 0.001 & 0.0001 & 0.0217 & 0.0007 \\
\hline \multirow{2}{*}{ Inconel 625 } & $\mathrm{Ni}$ & $\mathrm{Cr}$ & $\mathrm{Mo}$ & $\mathrm{Fe}$ & $\mathrm{Nb}$ & $\mathrm{Ti}$ \\
\cline { 2 - 7 } & 60.8 & 21.5 & 9.0 & 3.9 & 3.5 & 0.26 \\
\hline
\end{tabular}

625. The hydrogen content just after hydrogen charging was 1.3 mass ppm for pure iron and 87 mass ppm for Inconel 625.

Hydrogen desorption behavior during tensile deformation was analyzed using a slow strain rate technique in a vacuum chamber fitted with a rotary pump, turbomoleculer pump, quadrupole mass spectrometer (QMS), vacuum meter, and a baking heater. Specimens with/without hydrogen-charging were subjected to tensile stress until fracture or a cyclic elastic stress. Hydrogen desorption $(\mathrm{M} / \mathrm{z}=2)$ and water desorption $(\mathrm{M} / \mathrm{z}=18)$ from the specimens during tensile deformation were detected with the QMS in the vacuum chamber at room temperature at strain rates between $4.2 \times 10^{-6}$ and $4.2 \times 10^{-3} / \mathrm{s}$. The analysis was started about $20 \mathrm{~min}$ after hydrogen charging, when the vacuum pressure in the chamber was $5.0 \times 10^{-4} \mathrm{~Pa}$. The surfaces of hydrogen-charged specimens were polished with emery paper before the analysis because it was thought that hydrogen charging in the aqueous solution would increase the amount of absorbed water and cause surface unevenness.

Thermal desorption analysis (TDA) was conducted using a gas chromatograph at a constant heating rate of $100^{\circ} \mathrm{C} / \mathrm{h}$ to measure the amount of hydrogen desorbed during deformation. A standard gas mixture of $\mathrm{Ar}+50 \mathrm{vol} \mathrm{ppm}$ of $\mathrm{H}_{2}$ was used for the calibration of the hydrogen content at a sampling interval of $5 \mathrm{~min}$.

\section{Results}

\subsection{Deduction of Fragment Hydrogen Dissociated from $\mathrm{H}_{2} \mathrm{O}$}

Background hydrogen must be deducted in order to detect only hydrogen desorption promoted by tensile stress. Hydrogen desorbed from the specimen surface or from the chamber wall by heating had little influence in this experiment because the specimens were not heated. However, the influence of fragment hydrogen could not be ignored when considerable $\mathrm{H}_{2} \mathrm{O}$ was adsorbed physically on the specimen surface and/or on the chamber wall. Hydrogen $(\mathrm{M} / \mathrm{z}=2)$ and water $(\mathrm{M} / \mathrm{z}=18)$ desorption spectra and the corresponding stress-strain curve of a hydrogen-uncharged pure iron specimen annealed at $900^{\circ} \mathrm{C}$ and subjected to tensile stress at a strain rate of $4.2 \times 10^{-4} / \mathrm{s}$ until fracture are shown in Figs. 1(a) and 1(b). Although the hydrogen content in the hydrogen-uncharged specimen was found by TDA to be 0 mass ppm, a little hydrogen was detected as shown in Fig. 1(a). This hydrogen desorption spectrum is probably not for hydrogen from the specimen but for fragment hydrogen dissociated from $\mathrm{H}_{2} \mathrm{O}$ on the surfaces.

The ratio of fragment hydrogen desorption to the quantity of water desorption, which depends on the kind of QMS used, should be constant. This ratio is shown in Fig. 2 for the hydrogen-uncharged pure iron specimen subjected to tensile stress at a strain rate of $4.2 \times 10^{-4} / \mathrm{s}$ until fracture 

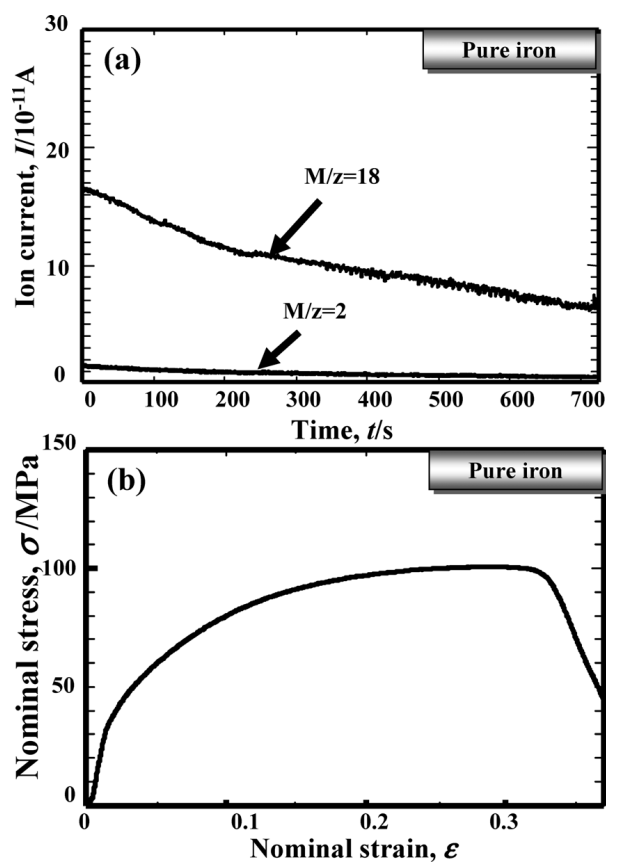

Fig. 1. Correlation between (a) desorbed hydrogen and water spectra and (b) stress-strain curve of a hydrogen-uncharged pure iron specimen during tensile deformation at a strain rate of $4.2 \times 10^{-4} / \mathrm{s}$.

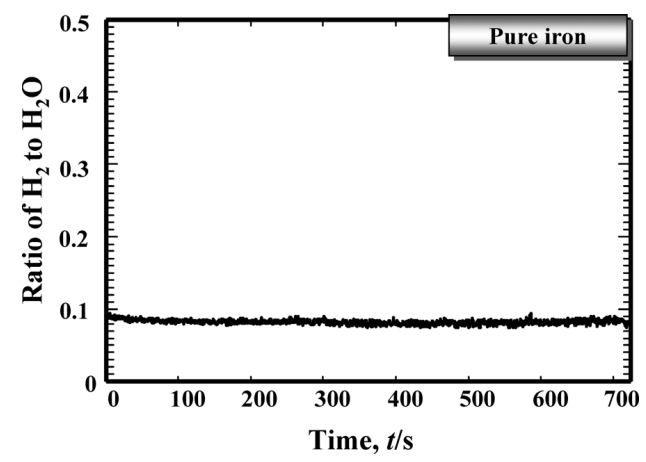

Fig. 2. Ratio of fragment hydrogen to the quantity of water desorbed from a hydrogen-uncharged pure iron specimen during tensile deformation at a strain rate of $4.2 \times 10^{-4} / \mathrm{s}$.

occurred. The ratio remained constant during tensile deformation until fracture. Therefore, this hydrogen desorption spectrum is not for hydrogen from inside the specimen but for fragment hydrogen dissociated from $\mathrm{H}_{2} \mathrm{O}$ and must be deducted as background. The ratio of hydrogen desorption to the quantity of water desorption for a hydrogen-uncharged Inconel 625 specimen was also constant during tensile deformation until fracture. This background hydrogen was deducted from the results described below.

\subsection{Diffusible Hydrogen Desorbed without Tensile De- formation}

The hydrogen desorption spectrum of a hydrogencharged pure iron specimen that was not subjected to tensile stress in the vacuum chamber is shown in Fig. 3. A little hydrogen desorbed from the specimen at room temperature, but no pronounced hydrogen desorption peak was observed. This hydrogen desorption resulted from the diffusion of hydrogen atoms in the lattice at room temperature by a thermally activated process. This diffusion release

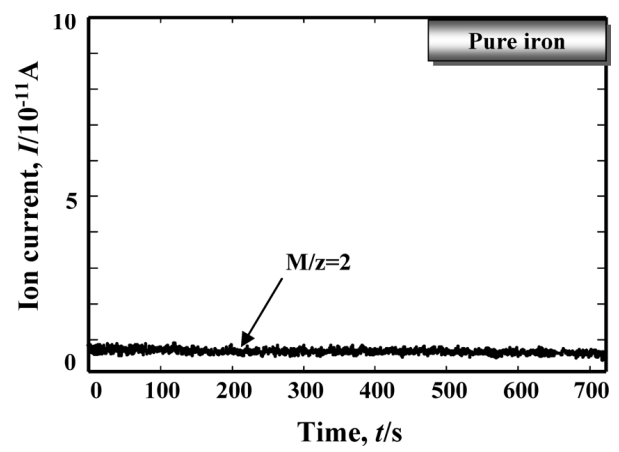

Fig. 3. Hydrogen desorption spectrum from a hydrogen-charged pure iron specimen without tensile deformation.

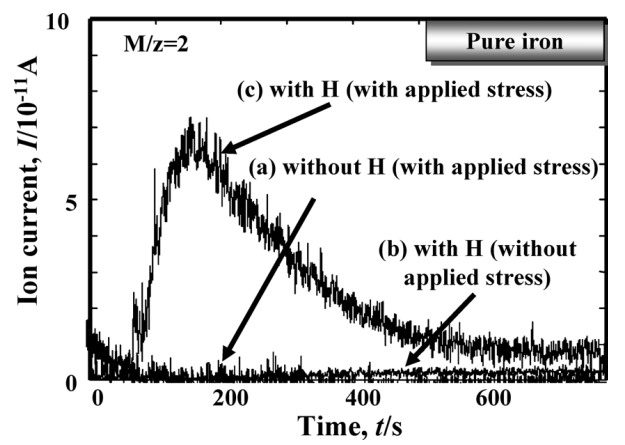

Fig. 4. Hydrogen desorption spectra from pure iron specimens: (a) without hydrogen-charging with tensile deformation at a strain rate of $4.2 \times 10^{-4} / \mathrm{s}$, (b) with hydrogen-charging without tensile deformation, and (c) with hydrogencharging with tensile deformation at a strain rate of $4.2 \times 10^{-4} / \mathrm{s}$.

without tensile stress must be deducted in order to discriminate only the hydrogen desorption promoted by tensile stress. Hydrogen desorption resulting from diffusion release in Inconel 625 was similar to that for pure iron. This diffusion release without tensile stress was deducted from the results described below.

\subsection{Determination of Hydrogen Desorption Ascrib- able to Tensile Stress}

On the basis of the foregoing discussion in Secs. 3.1 and 3.2 , hydrogen desorption promoted by tensile stress was determined by deducting the following two kinds of hydrogen from the total hydrogen desorption: (1) the fragment hydrogen dissociated from $\mathrm{H}_{2} \mathrm{O}$ on the specimen surface and/or on the chamber wall and (2) diffusive hydrogen desorbed without tensile stress at room temperature.

Figure 4 shows the hydrogen desorption spectra of (a) a hydrogen-uncharged pure iron specimen subjected to tensile stress at a strain rate of $4.2 \times 10^{-4} / \mathrm{s}$ until fracture, (b) a hydrogen-charged pure iron specimen without tensile stress, and (c) a hydrogen-charged pure iron specimen subjected to tensile stress at a strain rate of $4.2 \times 10^{-4} / \mathrm{s}$ until fracture. These hydrogen desorption spectra are the results after deducting both (1) and (2) above from the total hydrogen desorption. The results for (a) and (b) show no hydrogen desorption spectra, indicating that the background hydrogen desorption for both (1) and (2) was deducted from the total hydrogen desorption. It indicates that only the hydrogen desorption promoted by tensile stress was extracted correctly from the total hydrogen desorption. For Inconel 
625 as well, only hydrogen desorption promoted by tensile stress was extracted by deducting both (1) and (2) from the total hydrogen desorption.

\subsection{Hydrogen Desorption Behavior during Elastic/ Plastic Deformation}

Figure 5 shows (a) the hydrogen desorption spectrum and (b) the corresponding stress-strain curve of a hydrogencharged pure iron specimen subjected to tensile stress at a strain rate of $4.2 \times 10^{-4} / \mathrm{s}$ until fracture. Hydrogen desorption was small under elastic deformation. In contrast, it increased rapidly at the strain beyond the proof stress when plastic deformation began, reached its maximum, and then decreased gradually with increasing plastic strain.

Figure 6 shows (a) the hydrogen desorption spectrum and (b) the corresponding stress-strain curve of a hydrogencharged Inconel 625 specimen subjected to tensile stress at a strain rate of $4.2 \times 10^{-4} / \mathrm{s}$ until fracture. The hydrogen desorption behavior was similar to that of the pure iron specimen. It was markedly small under elastic deformation. In contrast, it increased rapidly at the strain beyond the proof stress when plastic deformation began, reached its maximum, decreased gradually as plastic strain increased, and then a sharp peak appeared at fracture. The sharp peak at fracture presumably indicates that hydrogen accumulating in the fracture area desorbs in a burst when the specimen fractures and then a fresh surface is exposed. In this experiment, hydrogen desorption did not decrease to zero after fracture. This is attributed to the change in vacuum pressure or the change in background hydrogen as a result of hydrogen desorption from the specimens during tensile testing. Hydrogen desorption after fracture was also observed in the results described below and the reason for it was probably the same.

To examine more closely the effect of elastic/plastic de-
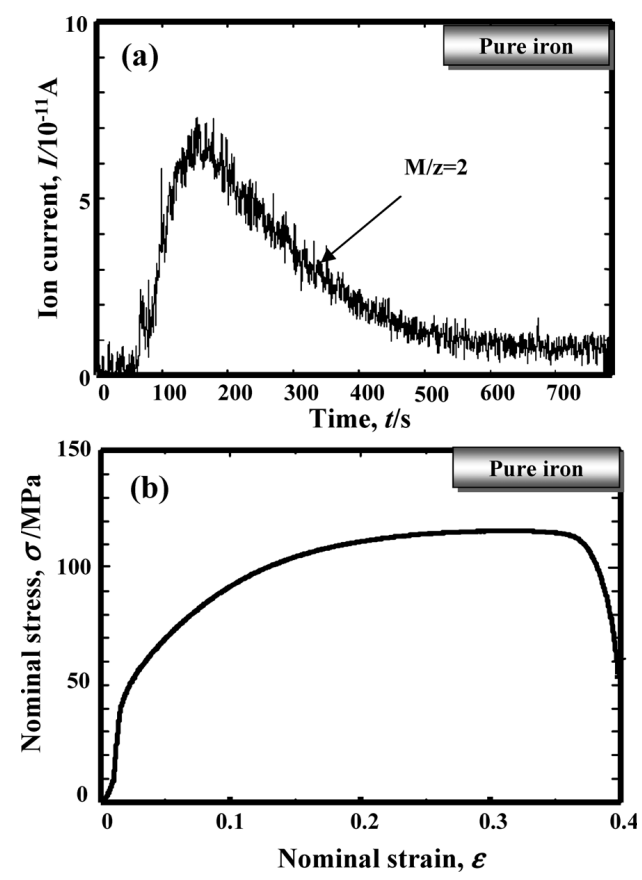

Fig. 5. Correlation between (a) hydrogen desorption spectrum and (b) stress-strain curve of a hydrogen-charged pure iron specimen during tensile deformation at a strain rate of $4.2 \times 10^{-4} / \mathrm{s}$. formation on hydrogen desorption behavior, cyclic elastic stress was applied prior to the final fracture. Figure 7 shows (a) the hydrogen desorption spectrum and (b) the corresponding stress-time curve of a hydrogen-charged pure iron specimen subjected to 30 elastic stress cycles between 20 and $40 \mathrm{MPa}$, followed by the application of tensile stress at a strain rate of $4.2 \times 10^{-4} / \mathrm{s}$ until fracture. Hydrogen desorption was small under cyclic elastic stress. It in-
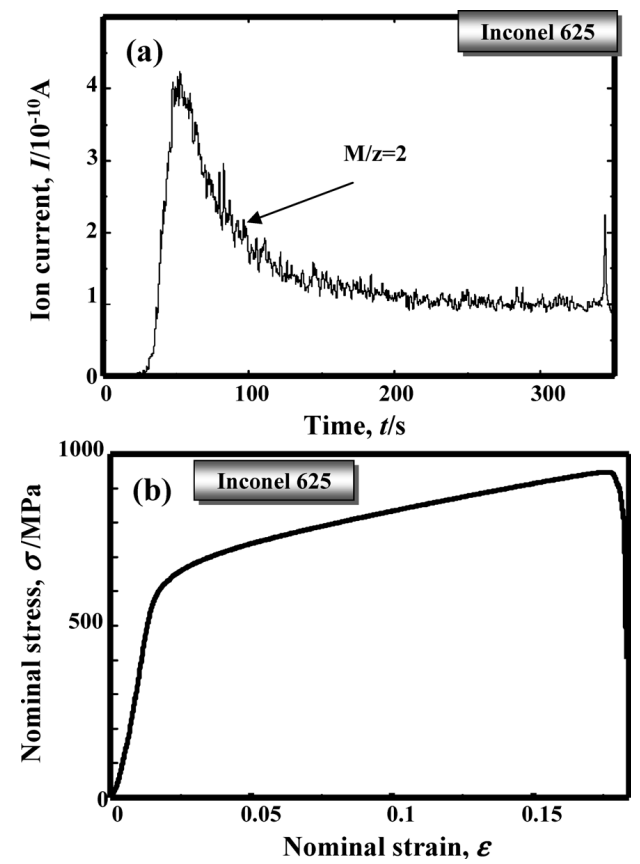

Fig. 6. Correlation between (a) hydrogen desorption spectrum and (b) stress-strain curve of a hydrogen-charged Inconel 625 specimen during tensile deformation at a strain rate of $4.2 \times 10^{-4} / \mathrm{s}$.
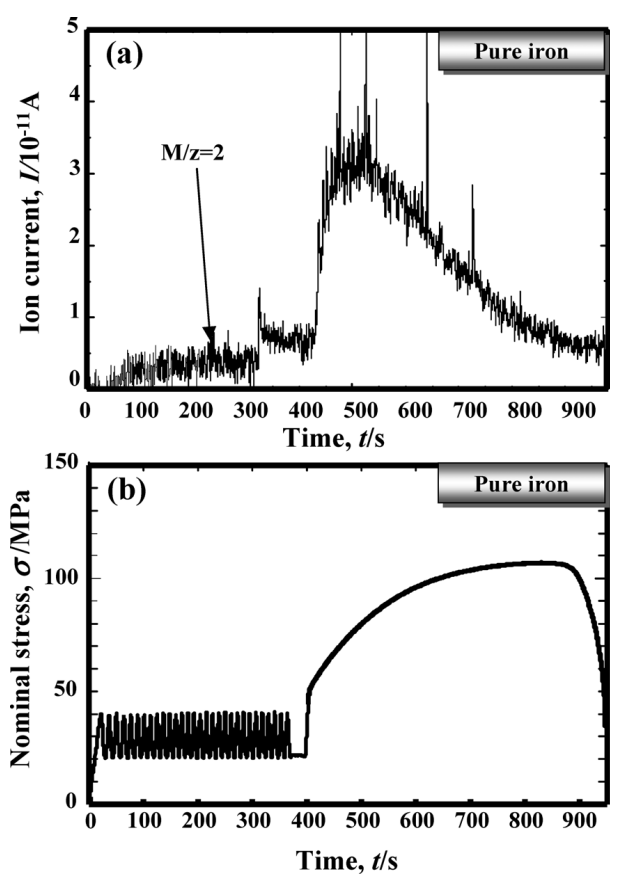

Fig. 7. Correlation between (a) hydrogen desorption spectrum and (b) stress-time curve of a hydrogen-charged pure iron specimen subjected to cyclic elastic stress and subsequent tensile stress until failure at a strain rate of $4.2 \times 10^{-4} / \mathrm{s}$. 

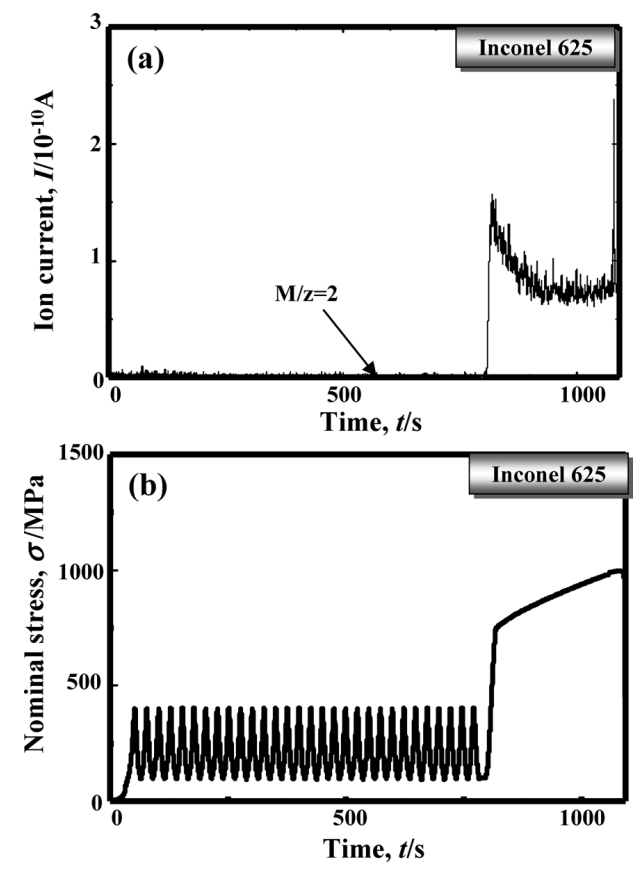

Fig. 8. Correlation between (a) hydrogen desorption spectrum and (b) stress-time curve of a hydrogen-charged Inconel 625 specimen subjected to cyclic elastic stress and subsequent tensile stress until failure at a strain rate of $4.2 \times 10^{-4} / \mathrm{s}$.

creased rapidly at the strain beyond the proof stress when plastic deformation began, reached its maximum, and then decreased gradually as plastic strain increased.

Figure 8 shows (a) the hydrogen desorption spectrum and (b) the corresponding stress-time curve of a hydrogencharged Inconel 625 specimen subjected to 30 elastic stress cycles between 100 and $400 \mathrm{MPa}$, followed by the application of tensile stress at a strain rate of $4.2 \times 10^{-4} / \mathrm{s}$ until fracture. Hydrogen desorption did not increase under cyclic elastic stress. It increased rapidly at the strain beyond the proof stress when plastic deformation began, reached its maximum, decreased gradually with increasing plastic strain, and then a sharp peak appeared at fracture.

\subsection{Strain Rate Dependence of the Amount of Des- orbed Hydrogen}

The hydrogen desorption spectra of hydrogen-charged specimens subjected to tensile stress at a strain rate of $4.2 \times 10^{-5} / \mathrm{s}$ or $4.2 \times 10^{-3} / \mathrm{s}$, i.e., ten times or one-tenth the rate used in Fig. 5(a) and Fig. 6(a), respectively, until fracture are shown in Figs. 9 and 10. In all the spectra, hydrogen desorption increased rapidly at the proof stress when plastic deformation began, reached its maximum, and then decreased gradually with increasing plastic strain.

Next, the amounts of desorbed hydrogen at various strain rates until fracture are compared. The amount of desorbed hydrogen cannot be measured exactly from the hydrogen desorption spectra during tensile deformation, since hydrogen desorption does not decrease to zero after fracture because of the change in background hydrogen as mentioned in Sec. 3.4. Hence, the amount of desorbed hydrogen was determined precisely by TDA calibrated using the hydrogen standard gas.

Table 2 shows the hydrogen contents of pure iron and In-
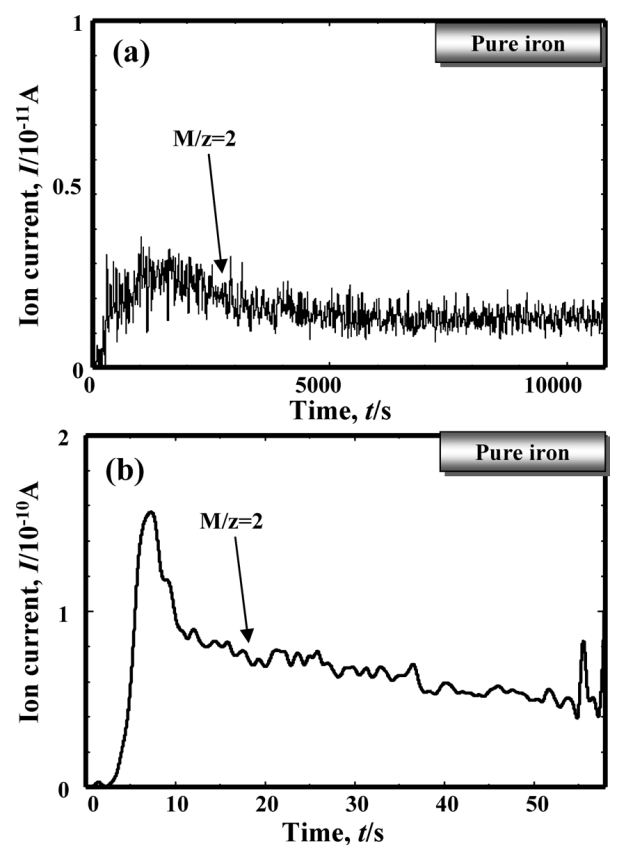

Fig. 9. Hydrogen desorption spectra from hydrogen-charged pure iron specimens during tensile deformation at strain rates of (a) $4.2 \times 10^{-5} / \mathrm{s}$ and (b) $4.2 \times 10^{-3} / \mathrm{s}$.
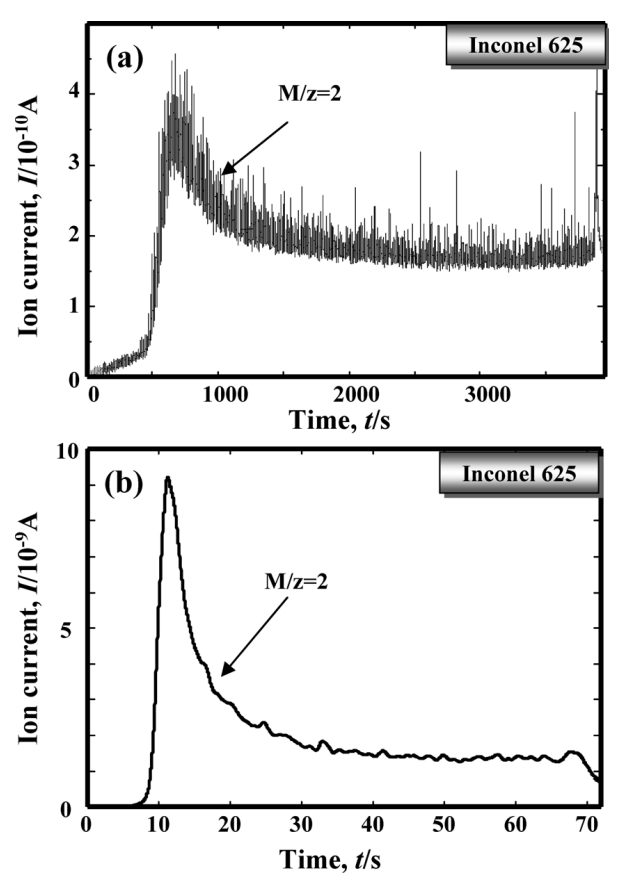

Fig. 10. Hydrogen desorption spectra from hydrogen-charged Inconel 625 specimens during tensile deformation at strain rates of (a) $4.2 \times 10^{-5} / \mathrm{s}$ and (b) $4.2 \times 10^{-3} / \mathrm{s}$.

conel 625 measured by TDA: (a) after tensile fracture at various strain rates, (b) after holding in the vacuum chamber without tensile stress for a duration corresponding to the time to fracture, and (c) the amount of desorbed hydrogen ((b)-(a)) promoted by tensile deformation until fracture and the ratio of desorbed hydrogen to the initial content. The strain rates used were $4.2 \times 10^{-5} / \mathrm{s}, 4.2 \times 10^{-4} / \mathrm{s}$ and $4.2 \times 10^{-3} / \mathrm{s}$ for pure iron and $4.2 \times 10^{-6} / \mathrm{s}, 4.2 \times 10^{-5} / \mathrm{s}$, $4.2 \times 10^{-4} / \mathrm{s}$ and $4.2 \times 10^{-3} / \mathrm{s}$ for Inconel 625 . The percentages in parentheses in Table 2 are the ratio of the desorbed hydrogen content to the initial hydrogen content. For pure 
Table 2. Hydrogen contents of pure iron and Inconel 625 (a) after tensile failure at various strain rates, (b) after holding in the vacuum chamber without undergoing tensile test for the time corresponding to the time to fracture, and (c) desorbed hydrogen contents ((b)-(a)) promoted by tensile deformation until failure and the ratio of desorbed hydrogen to the initial content.

\begin{tabular}{|c|c|c|c|c|c|}
\hline \multicolumn{2}{|c|}{ Hydrogen content (mass ppm) } \\
\hline \multicolumn{2}{|c|}{ Strain rates } & $4.2 \times 10^{-6} / \mathrm{s}$ & $4.2 \times 10^{-5} / \mathrm{s}$ & $4.2 \times 10^{-4} / \mathrm{s}$ & $4.2 \times 10^{-3} / \mathrm{s}$ \\
\hline \multirow{3}{*}{ Pure iron } & (a) H after failure & - & 0.13 & 0.70 & 1.25 \\
\cline { 2 - 7 } & (b) H after holding time in vacuum & - & 0.23 & 0.91 & 1.30 \\
\cline { 2 - 7 } & (c) H promoted by tensile deformation & - & 0.10 & 0.21 & 0.05 \\
& and ratio of H & & $(8 \%)$ & $(16 \%)$ & $(4 \%)$ \\
\hline \multirow{4}{*}{ Inconel 625 } & (a) H after failure & 61.2 & 70.8 & 85.6 & 86.4 \\
\cline { 2 - 7 } & (b) H after holding time in vacuum & 68.7 & 75.3 & 86.1 & 86.6 \\
\cline { 2 - 7 } & (c) H promoted by tensile deformation & 7.5 & 4.5 & 0.5 & 0.2 \\
& and ratio of H & $(9 \%)$ & $(5 \%)$ & $(1 \%)$ & $(0 \%)$ \\
\hline
\end{tabular}

iron, the largest amount of desorbed hydrogen promoted by tensile stress was 0.21 mass ppm, i.e., $16 \%$ of the initial hydrogen content, when the specimen was deformed at a strain rate of $4.2 \times 10^{-4} / \mathrm{s}$. In contrast, the amount of desorbed hydrogen promoted by tensile stress increased with a decreasing strain rate for Inconel 625 and was $9 \%$ of the initial hydrogen content when the specimen was deformed at a strain rate of $4.2 \times 10^{-6} / \mathrm{s}$. For both specimens, the amount of desorbed hydrogen promoted by tensile deformation depended on the stain rate.

\section{Discussion}

\subsection{Factors Promoting Hydrogen Desorption during Tensile Deformation}

Figures 5 to 10 show that the hydrogen ion intensity (vertical axes) after tensile deformation did not shift to the negative side (restraint of hydrogen desorption) but acted on the positive side (promotion of hydrogen desorption), based on the amount of hydrogen desorbed before tensile deformation. These results indicate that hydrogen desorption is promoted by tensile stress even at room temperature. Moreover, hydrogen desorption was small under elastic deformation, whereas it increased rapidly when plastic deformation began. In this section, the factors promoting hydrogen desorption by deformation are discussed.

The following five factors are presumed to promote hydrogen desorption by tensile stress: (A) an increase in the specimen temperature caused by heat generated during deformation, (B) fragment hydrogen dissociated from $\mathrm{H}_{2} \mathrm{O}$ adsorbed on the specimen surface, $(\mathrm{C})$ the fracture of the oxide film on the specimen surface, (D) an uneven specimen surface formed by deformation (increase in specimen surface area), and (E) hydrogen transport by moving dislocations.

The following two factors are presumed to restrain hydrogen desorption by tensile stress: (F) an increase in hydrogen trapping sites, i.e., lattice defects, created by plastic deformation and $(\mathrm{G})$ an increase in hydrogen absorption capacity due to a decrease in chemical potential. ${ }^{26)}$

In this study, the restraining effect of factor (F) occurred naturally because of the increase in lattice defects such as dislocations and vacancies that acted as trapping sites during plastic deformation. However, it is clear that some of the factors promoting hydrogen desorption exceeded the factors restraining it. On the basis of the strain rate dependence of hydrogen desorption, the factors (A) to (E) promoting hydrogen desorption by tensile stress are discussed here for the purpose of showing which one is the most effective.

First, factor (A) concerning an increase in the specimen temperature due to heat generated by deformation can be ignored. This is because the amount of desorbed hydrogen increased with a decreasing strain rate for Inconel 625, although the specimen temperature usually increases with the strain rate. Actual measurement of the specimen surface temperature during tensile testing indicated that it increased $2{ }^{\circ} \mathrm{C}$ until fracture at a strain rate of $4.2 \times 10^{-3} / \mathrm{s}$, but no change in the temperature was detected at a strain rate of $4.2 \times 10^{-5} / \mathrm{s}$. Secondly, factor (B) concerning fragment hydrogen dissociated from $\mathrm{H}_{2} \mathrm{O}$ adsorbed on the specimen surface can also be ignored. This is because hydrogen desorption was not observed for the former specimen shown in Fig. 4(a), although the specimen surface conditions were same for both the hydrogen-uncharged and hydrogencharged specimens as a result of polishing the latter specimen surfaces before the analysis. Thirdly, factor (C) concerning the fracture of the oxide film on the specimen surface and factor (D) concerning an uneven specimen surface formed by deformation (increase in specimen surface area) had little effect on hydrogen desorption. This is because the amount of desorbed hydrogen tended to depend on the strain rate, although the given fracture strains were almost the same and were large for all the strain rates. Finally, the moving velocity of dislocations, i.e., strain rate, is probably related to hydrogen transport by moving dislocations. The relationship between the amount of desorbed hydrogen and the strain rates can be seen in Table 2. Therefore, factor (E) concerning hydrogen transport by moving dislocations is concluded to be the most effective factor promoting hydrogen desorption by tensile stress.

\subsection{Relationship between Hydrogen Transport by Moving Dislocations and Stress-Strain Curve}

Hydrogen desorption during tensile deformation is thought to be closely related to hydrogen transport by moving dislocations. In this section, the relationship between the hydrogen desorption spectrum and stress-strain curve is discussed.

Hydrogen desorption behavior during elastic deformation is discussed first. As shown in Figs. 6, 8 and 10, hydro- 
gen desorption during elastic deformation was not detected for Inconel 625 with its high proof stress and distinct boundary between elastic and plastic deformation. This behavior implies that dislocations do not move in Inconel 625, so hydrogen transport by moving dislocations does not occur. The chemical potential of Inconel 625 may be reduced by elastic tensile stress (factor $(\mathrm{G})$ ), which acts to restrain hydrogen desorption. In contrast, for pure iron with its low proof stress and without an obvious yield point, slight hydrogen desorption during elastic deformation was detected as shown in Figs. 5, 7 and 9. This is because the pinning of dislocations in pure iron does not occur, allowing mobile dislocations on slip lines to move with hydrogen and to transport hydrogen to the specimen surface in spite of the elastic stress.

Next, we will discuss hydrogen desorption behavior during plastic deformation. In both metals, hydrogen desorption increased rapidly at the strain beyond proof stress when plastic deformation began, reached its maximum, and then decreased gradually as plastic strain increased. This implies that hydrogen desorption during plastic deformation corresponds to the moving dislocations. The amount of hydrogen transported by moving dislocations at a constant strain rate depends on the concentration of hydrogen around dislocations and the amount of moving dislocations reaching the specimen surface. The concentration of hydrogen around dislocations is expressed as follows ${ }^{27)}$ :

$$
C_{x}=C_{L} \exp \left(E_{B} / k_{B} T\right)
$$

where $C_{x}$ is the concentration of hydrogen by atomic ratio at trapping sites, $C_{L}$ is the concentration of solid solution hydrogen, $k_{B}$ is the Boltzmann constant, $T$ is absolute temperature, and $E_{B}$ is the binding energy between trapping sites and hydrogen. Equation (1) indicates that the concentration of hydrogen around dislocations depends on $E_{B}$ and $T$, when the initial hydrogen concentration is constant. As tensile tests were conducted in this study at a constant temperature, the concentration of hydrogen around dislocations depends on only $E_{B}$. In $\alpha$-iron with a bcc lattice, the interaction between edge dislocations with a hydrostatic stress field and hydrogen is generally large, and the binding energy between edge dislocations and hydrogen is reported to be $58 \mathrm{~kJ} / \mathrm{mol}^{28)}$ In contrast, the interaction between screw dislocations without a hydrostatic stress field and hydrogen is smaller than that of edge dislocations. Thus, the binding energy between screw dislocations and hydrogen is reported to be $19 \mathrm{~kJ} / \mathrm{mol}^{28)}$ and $20-30 \mathrm{~kJ} / \mathrm{mol}^{29)}$ Although the binding energy values differ among researchers, there is agreement that the binding energy between edge dislocations and hydrogen is larger than that of screw dislocations. Accordingly, the concentration of hydrogen around edge dislocations is greater than that around screw dislocations as expressed in Eq. (1), which means that a Cottrell atmosphere of hydrogen easily forms around edge dislocations, and a high concentration of hydrogen forms at the core of edge dislocations in bcc metals.

The binding energy between dislocations and hydrogen is $8.7-19.3 \mathrm{~kJ} / \mathrm{mol}^{30)}$ in pure nickel with an fcc lattice. This level of binding energy is much lower than that of bcc metals and it suggests that the concentration of hydrogen around dislocations in fcc metals is smaller than that for bcc metals. For example, it is reported that the hydrogen diffusion rate decreases with increasing dislocation density in $2.25 \mathrm{Cr}-1 \mathrm{Mo}$ steel with a bcc lattice. ${ }^{31)}$ This indicates that the interaction between hydrogen and dislocations in bcc metals is large. In contrast, the hydrogen diffusion rate increases due to plastic deformation in pure Ni with an fcc lattice. ${ }^{32)}$ This means that the interaction between hydrogen and dislocations in fcc metals is little connected with hydrogen diffusion. These findings show the difference in the interaction between dislocations and hydrogen in bec metals and that between dislocations and hydrogen in fcc metals.

Next, we will consider the relationship between hydrogen transport by moving dislocations and the stress-strain curve. In general, edge dislocation mobility is larger than that of screw dislocations at the beginning of plastic deformation in every crystal structure under room temperature. ${ }^{11)}$ Therefore, edge dislocations with high mobility move on the slip plane and reach the specimen surface first, and then screw dislocations play a main role in subsequent plastic deformation. This difference in mobility between edge dislocations and screw dislocations corresponds to the hydrogen desorption spectra obtained in the present study. The sharp increase in hydrogen desorption when plastic deformation begins implies that much hydrogen is transported to the specimen surface by edge dislocations having large binding energy with hydrogen. The dislocation density is low and there are few obstacles on the slip plane at the onset of plastic deformation, so dislocations can move straight and over a long distance on the slip plane of the primary slip system. The decrease in hydrogen desorption during subsequent plastic deformation corresponds to the decrease in hydrogen transport by screw dislocations. This is because the binding energy between screw dislocations and hydrogen is smaller, i.e., the Cottrell atmosphere of hydrogen is smaller around screw dislocations. In addition, the number of dislocations moving over a long distance to the specimen surface decreases, since their intersections tend to cause cross slips, resulting in the formation of a tangled cell structure, and pile-ups at grain boundaries. Furthermore, the density of vacancies during subsequent plastic deformation is greater than that at the beginning of plastic deformation. Since vacancies are more stable trapping sites for hydrogen than dislocations, ${ }^{13)}$ hydrogen tends to be trapped at vacancies in the specimen and therefore the amount of hydrogen transported by moving dislocations may decrease.

\subsection{Relationship between Hydrogen Transported by Moving Dislocations and Strain Rate}

In the preceding section, the factors affecting hydrogen transport by moving dislocations at a constant strain rate were discussed. The strain rate dependence of hydrogen transport by moving dislocations can be seen in the results shown Table 2. This section discusses the effect of the strain rate on hydrogen transport by moving dislocations.

The detrapping of hydrogen and the presence of hydrogen at the interstitial position in the lattice without trapping increase with temperature as shown in Eq. (1). Therefore, the fraction of hydrogen present at dislocation cores and around the elastic stress field of dislocations decreases with increasing temperature. Even if many dislocations move on the slip plane and reach the specimen surface, the strain 
rate has little effect on the amount of hydrogen transported by moving dislocations at higher temperature because of the low hydrogen concentration around dislocations. In contrast, the effect of the strain rate (average dislocation velocity) is larger at lower temperature because of the higher concentration of hydrogen around dislocations.

The average dislocation velocity $v$ is expressed as ${ }^{11)}$

$$
v=2 \dot{\varepsilon} / \rho b
$$

where $\dot{\varepsilon}$ is the strain rate, $\rho$ is the density of mobile dislocations and $b$ is the Burgers vector. When the dislocation velocity $v$ and the hydrogen diffusion rate are the same or $v$ is slightly bigger, hydrogen can move with moving dislocations and the interaction between dislocations and hydrogen has the maximum effect. For pure iron, the largest amount of desorbed hydrogen promoted by tensile stress was 0.21 mass ppm, i.e., $16 \%$ of the initial hydrogen content, when the metal was deformed at a strain rate of $4.2 \times$ $10^{-4} / \mathrm{s}$. This result indicates that the interaction between dislocations and hydrogen had a sufficient effect at this strain rate. The reason for the decrease in the amount of hydrogen transported by moving dislocations at a strain rate of $4.2 \times 10^{-3} / \mathrm{s}$, i.e., ten times higher than $4.2 \times 10^{-4} / \mathrm{s}$, is that the dislocation velocity was much higher than the hydrogen diffusion rate. As a result, only a little hydrogen interacted with dislocations. The reason for the decrease in the amount of hydrogen transported by moving dislocations at a strain rate of $4.2 \times 10^{-5} / \mathrm{s}$, i.e., one-tenth higher than $4.2 \times 10^{-4} / \mathrm{s}$, is that the hydrogen diffusion rate was much higher than the dislocation velocity. Therefore, hydrogen self-diffused to the specimen surface by a thermally activated process before it was transported by dislocations.

The hydrogen diffusion coefficient in Inconel 625 is six orders of magnitude lower than that in pure iron at room temperature. ${ }^{33)}$ Hence, the average dislocation velocity is much higher than the hydrogen diffusion velocity at a strain rate of $4.2 \times 10^{-4} / \mathrm{s}$ for Inconel 625 . It is expected that the interaction between dislocations and hydrogen can occur when the strain rate is three orders of magnitude lower than $4.2 \times 10^{-4} / \mathrm{s}$. The amount of desorbed hydrogen promoted by tensile stress was $1 \%, 5 \%$ and $9 \%$ of the initial hydrogen content at strain rates of $4.2 \times 10^{-4} / \mathrm{s}, 4.2 \times 10^{-5} / \mathrm{s}$ and $4.2 \times 10^{-6} / \mathrm{s}$, respectively, as shown in Table 2 . These experimental results concerning the strain rate dependence of hydrogen desorption agree well with the expected interaction between dislocations and hydrogen velocities. Hydrogen desorption increased when the average dislocation velocity decreased. As discussed in the preceding section, three reasons can be considered for the higher ratio of hydrogen transport by moving dislocations seen for pure iron (16\%) than for Inconel 625. (I) The binding energy between dislocations and hydrogen is greater in bcc metals than in fcc metals. (II) Hydrogen stable sites in iron with a bcc lattice are tetrahedral sites on the slip plane (112) of bcc metals, allowing hydrogen to be dragged easily by moving dislocations. (III) Dislocations can move over a long distance in pure iron.

The results of the present study using a pure iron and Inconel 25 have made it clear that hydrogen is transported by moving dislocations, which is one of the physical phenomena occurring under hydrogen and stress conditions. Hydro- gen desorption promoted by tensile stress does not cause hydrogen embrittlement directly, but it is an important elemental phenomenon involved in such embrittlement with respect to the increased creation of lattice defects by dislocation dynamics enhanced by hydrogen and hydrogen accumulation in local areas such as at grain boundaries, cell walls, various precipitates and inclusions. Hydrogen desorption behavior in high-strength steels with their many barriers to dislocation mobility such as high dislocation densities, numerous kinds of precipitates and refined grain boundaries needs to be examined further in the context of hydrogen embrittlement.

\section{Conclusions}

The desorption behavior of weakly trapped diffusive hydrogen, causing a reduction of fracture strain, has been analyzed in situ during deformation at room temperature using a pure iron as a bcc metal and an Inconel 625 alloy as an fcc metal. The results obtained regarding the effect of elastic/plastic deformation on hydrogen desorption behavior can be summarized as follows.

(1) Hydrogen desorption promoted by tensile stress can be estimated by deducting both fragment hydrogen dissociated from adsorbed $\mathrm{H}_{2} \mathrm{O}$ and $\mathrm{H}_{2}$ desorbed without applying stress at room temperature from the total amount of hydrogen desorbed from hydrogen-charged specimens during tensile deformation.

(2) Hydrogen desorption seldom increases under elastic deformation. In contrast, it increases rapidly after the proof stress when plastic deformation begins, reaches its maximum, and then decreases gradually with increasing applied strain. This desorption behavior is probably closely related to moving dislocations during plastic deformation and depends on the dislocation type such as edge or screw dislocations and dislocation mobility.

(3) The amount of desorbed hydrogen during tensile deformation differs at different strain rates. The largest amount of desorbed hydrogen promoted by tensile stress was observed when pure iron with a high hydrogen diffusion coefficient was deformed at a strain rate of $4.2 \times$ $10^{-4} / \mathrm{s}$. It was $16 \%$ of the initial hydrogen content. The amount of desorbed hydrogen decreased at strain rates of $4.2 \times 10^{-5} / \mathrm{s}$ and $4.2 \times 10^{-3} / \mathrm{s}$. In contrast, the amount of desorbed hydrogen increased with a decreasing strain rate for Inconel 625 with a lower hydrogen diffusion coefficient. The largest amount of desorbed hydrogen promoted by tensile stress was $9 \%$ of the initial hydrogen content when Inconel 625 was deformed at a strain rate of $4.2 \times 10^{-6} / \mathrm{s}$. This difference in the amount of desorbed hydrogen is probably related to the amount of hydrogen that can move with moving dislocations.

\section{Acknowledgements}

The present study was conducted as part of research activities for "Fundamental Studies on Technologies for Steel Materials with Enhanced Strength and Functions" promoted by the Consortium of The Japan Research and Development Center of Metals (JRCM). Financial support from the New Energy and Industrial Technology Development Organization (NEDO) is gratefully acknowledged. In 
addition, part of this study was supported by a Grant-in-Aid for Science Research B (No. 18360336). The authors wish to express their thanks to Nobuyoshi Miyabayashi and $\mathrm{Ku}-$ nimitsu Maejima at ESCO, Ltd. for their valuable advice about hydrogen desorption spectra.

\section{REFERENCES}

1) K. Takai and R. Watanuki: ISIJ Int., 43 (2003), 520.

2) K. Takai, G. Yamauchi, M. Nakamura and M. Nagumo: J. Jpn. Inst. Met., 62 (1998), 267.

3) K. Takai, Y. Chiba, K. Noguchi and A. Nozue: Metall. Mater. Trans. $A$, 33A (2002), 2659.

4) K. Takai and A. Nozue: J. Jpn. Inst. Met., 64 (2000), 669.

5) R. A. Oriani and P. H. Josephic: Acta Metall., 22 (1974), 1065.

6) H. K. Birnbaum and P. Sofronis: Mater. Sci. Eng., A176 (1994), 191

7) M. Nagumo: Mater. Sci. Technol., 20 (2004), 940.

8) S. P. Lynch: Acta Metall., 36 (1988), 2639.

9) K. Takai, H. Shoda, H. Suzuki and M. Nagumo: Acta Metall., 56 (2008), 5158

10) H. Shoda, H. Suzuki, K. Takai and Y. Hagihara: CAMP-ISIJ, 21 (2008), 1382, CD-ROM.

11) Y. Kohda: Introduction to Metal Physics, Corona Publishing, Tokyo, (1997), 213

12) K. Sakaki, T. Kawase, M. Hirato, M. Mizuno, H. Araki, Y. Shirai and M. Nagumo: Scr. Metall., 55 (2006), 1031.
13) Y. Tateyama and T. Ohono: ISIJ Int., 43 (2003), 573.

14) U. Essmann and H. Mughrab: Philos. Mag., A40 (1979), 731.

15) A. M. Cuitino and M. Ortiz: Acta Metall., 44 (1996), 427.

16) H. Hagi: J. Jpn. Inst. Met., 57 (1993), 864.

17) M. R. Louthan, G. R. Caskey, J. A. Donovan and D. E. Rawl: Mater. Sci. Eng., 10 (1972), 357

18) J. A. Donovan: Mater. Trans. A, 7A (1975), 1677.

19) A. M. Brass and J. Chene: Mater. Sci. Eng., A242 (1998), 210.

20) J. Chene and A. M. Brass: Scr. Metall., 40 (1999), 537.

21) A. M. Brass and J. Chene: Corros. Sci., 48 (2006), 3222.

22) K. Koyama, G. Itoh and M. Kanno: J. Jpn. Inst. Met., 62 (1998), 742.

23) K. Horikawa and K. Yoshida: J. Jpn. Inst. Met., 68 (2004), 1043.

24) M. Kanno, H. Okada and G. Itoh: J. Jpn. Inst. Met., 59 (1995), 296.

25) K. Takai, K. Murakami, N. Yabe, H. Suzuki and Y. Hagihara: J. Jpn. Inst. Met., 72 (2008), 448.

26) J. O'M. Bockris, W. Beck, M. A. Genshaw, P. K. Subramanya and F. S. Williams: Acta Metall., 19 (1971), 1209.

27) M. Nagumo: Zairyo-to-Kankyo, 54 (2005), 306.

28) C. Hwang and I. M. Bernstein: Acta Metall., 34 (1986), 1001.

29) J. P. Hirth: Metall. Trans. A, 11A (1980), 861.

30) G. J. Thomas: Hydrogen Effects in Metals, ed. by I. M. Bernstein and A. W. Thompson, AIME, Warrendale, (1981), 77.

31) M. Kurkela, G. S. Frankel and P. M. Latanission: Scr. Metall., 16 (1982), 455.

32) M. Kurkela and P. M. Latanission: Scr. Metall., 13 (1979), 927.

33) D. J. Fisher: Hydrogen Diffusion in Metals, Trans. Tech. Publications, Switzerland, (1998), 178. 